The University of Southern Mississippi

The Aquila Digital Community

Faculty Publications

$6-2018$

\title{
The Method of Particular Solutions Using Trigonometric Basis Functions
}

Zhaolu Tian

Taiyuan University of Technology

Xinxiang Li

Shanghai University

C.M. Fan

National Taiwan Ocean University

Ching-Shyang Chen

University of Southern Mississippi, cs.chen@usm.edu

Follow this and additional works at: https://aquila.usm.edu/fac_pubs

Part of the Mathematics Commons

\section{Recommended Citation}

Tian, Z., Li, X., Fan, C., Chen, C. (2018). The Method of Particular Solutions Using Trigonometric Basis Functions. Journal of Computational and Applied Mathematics, 335, 20-32.

Available at: https://aquila.usm.edu/fac_pubs/14925

This Article is brought to you for free and open access by The Aquila Digital Community. It has been accepted for inclusion in Faculty Publications by an authorized administrator of The Aquila Digital Community. For more information, please contact Joshua.Cromwell@usm.edu. 


\title{
The Method of Particular Solutions using Trigonometric Basis Functions
}

\author{
Zhao-Lu Tian, Xinxiang Li $\stackrel{\dagger}{\dagger}$ C.M. Fan§ C.S. Chen
}

\begin{abstract}
In this paper, the method of particular solutions (MPS) using trigonometric functions as the basis functions is proposed to solve two-dimensional elliptic partial differential equations. The inhomogeneous term of the governing equation is approximated by Fourier series and the closed-form particular solutions of trigonometric functions are derived using the method of undetermined coefficients. Once the particular solutions for the trigonometric basis functions are derived, the standard MPS can be applied for solving partial differential equations. In comparing with the use of radial basis functions and polynomials in the MPS, our proposed approach provide another simple approach to effectively solving two-dimensional elliptic partial differential equations. Five numerical examples are provided in this paper to validate the merits of the proposed meshless method.
\end{abstract}

Keywords: method of particular solutions, trigonometric functions, particular solution, meshless methods, collocation method

\section{Introduction}

During the past half century, the developments and applications of numerical schemes for boundary value problems have made significant progress. Various novel numerical methods have been proposed and successfully applied to realistic engineering applications. The finite difference method [1], the finite element method [2], the finite volume

${ }^{*}$ College of Data Science, Taiyuan University of Technology, Taiyuan, China

${ }^{\dagger}$ Department of Mathematics, Shanghai University, Shanghai, China

${ }^{\ddagger}$ Corresponding author: Xinxiang Li, E-mail: xinxiang.lee@t.shu.edu.cn

$\S$ Department of Harbor and River Engineering \& Computation and Simulation Center, National Taiwan Ocean University, Keelung 20224, Taiwan

『Department of Mathematics, University of Southern Mississippi, Hattiesburg, MS, 39406, U.S.A. 
method [1] and the boundary element method [3] are some of the most popular numerical methods and numerous commercial computer packages, based on these methods, are developed. Although these methods are popular with researchers and engineers, the tasks of meshing and numerical quadrature of these methods could sharply increase the human labor and computational cost. Thus, several so-called meshless (or meshfree) methods were proposed, such as the meshless local Petrov-Galerkin method [4], the element-free Galerkin method [4], the method of fundamental solutions (MFS) [5]-[7], the method of particular solutions (MPS) [8]-[9], the generalized finite difference method [10], the reproducing kernel particular method [11], etc. The MPS, adopted in this paper, is one of the newly-developed meshless methods and is truly free from time-consuming tasks of meshing the computational domain and numerical quadrature.

The MPS has been proven to be an effective method and easy for implementation. Recently, the MPS has been continuously improved and applied for solving various interesting problems. For example, Chen et al. [12] combined the adaptive greedy algorithm and the MPS to solve three-dimensional inhomogeneous elliptic equations. They used multiquadrics (MQ) function, one kind of radial basis functions (RBFs), to approximate inhomogeneous term and the adaptive greedy algorithm is responsible for alleviating the problems of ill-conditioning matrices and free parameters in the MQ function. In addition, two-dimensional linear elasticity problems and Navier-Stokes equations are efficiently and accurately solved using the MPS with MQ as the basis function [13, 14]. Furthermore, Jiang et al. [15] and Fu et al. [16] adopted the MPS to efficiently solve the inverse problem and fractional diffusion models, respectively. Besides, the localized MPS (LMPS) has been recently proposed by Yao et al. [17] for solving large-scale problems. The LMPS has been successfully applied to velocity-vorticity formulation of the NavierStokes equations by Fan et al. [18]. From the above discussions, it could be realized that the newly-developed MPS is getting matured and has great potential to be extended to solving real-life problems. In this paper we proposed a new basis function, which is the trigonometric function, to the MPS in order to alleviate the difficulty of choosing the shape parameter of RBFs.

In most of the MPS-related study, RBFs are the primary basis functions being used. Other than the RBFs as the basis function in the MPS, there are some other choices. In [19]-[20], the Chebyshev polynomials have been considered to be the basis functions in the MPS instead of the RBFs. Karageorghis and Kyza [19] adopted the Chebyshev polynomials to approximate the inhomogeneous term and used the MPS to accurately analyzed Poisson equation, inhomogeneous Helmholtz equation and inhomogeneous biharmonic equation. In order to ensure the spectral convergence of the Chebyshev polynomials, the spatial positions of collocation points should be placed at Gauss-Lobatto points in the interval $(-1,1)$. Therefore, extra attentions should be paid on the tasks of choosing collocation points and extrapolating the inhomogeneous term if the Chebyshev polynomials are used as the basis functions in the MPS. In addition to the RBFs and the Chebyshev polynomials, high-order polynomials are adopted as the basis functions in the MPS and 
its corresponding basis functions are derived by Dangal et al. [21]. The inhomogeneous term is expanded as a series of polynomials and then the particular solutions can be expressed as a series of corresponding polynomials. Even though the resultant system of linear algebraic equations is highly ill-conditioning, the multiple-scales technique [22] can be adopted to alleviate this difficulty and highly accurate solutions can be achieved.

The study in this paper is motivated by Dangal et al. [21], so we used the trigonometric functions as the basis functions in the MPS in addition to the RBFs, the Chebyshev polynomials, and the high-order polynomials. In our proposed approach, the Fourier series is adopted to approximate the inhomogeneous term of governing equation and then the particular solution can be expressed as a linear combination of trigonometric functions and products of trigonometric functions. The corresponding basis functions are derived in this paper when the trigonometric functions are adopted as the basis function in the MPS. The proposed trigonometric functions-based MPS is free from parameters in comparing with RBFs, has more flexibility in choosing collocation points in comparing with Chebyshev polynomials, and faces minor problem of ill-conditioning matrices in comparing with high-order polynomials. Two-dimensional constant-coefficients convection-diffusion equations are considered in this paper and five examples are provided to demonstrate the merits of the proposed approach.

This paper is organized as follows: review of relevant papers and discussions of motivation of this study are presented in the section of Introduction. The numerical procedures of the MPS and the mathematical derivations of the corresponding basis functions are given after the description of mathematical formulations of boundary value problems. Besides, the numerical results and comparisons of five examples are exhibited to show the characteristics of the proposed method. Some discussions, conclusions and possible future researches are drawn in the final section.

\section{Mathematical formulation of elliptic boundary value problem}

Let $\Omega$ be the computational domain and $\partial \Omega$ its boundary. In this paper, we consider twodimensional boundary value problem governed by elliptic partial differential equation,

$$
\mathcal{L} u(x, y)=f(x, y), \quad(x, y) \in \Omega,
$$

where

$$
\mathcal{L}=\left(\nabla^{2}+\alpha \frac{\partial}{\partial x}+\beta \frac{\partial}{\partial y}+\gamma\right)
$$

$\alpha, \beta$, and $\gamma$ are constant coefficients, and $f(x, y)$ is a known function. In addition to the governing equation, the solution, $u(x, y)$, should satisfy some well-posed boundary 
conditions along the whole boundary. In this paper, both of Dirichlet and Neumann boundary conditions are considered:

$$
u(x, y)=g_{1}(x, y), \quad(x, y) \in \Gamma^{D}
$$

and

$$
\frac{\partial u}{\partial n}=g_{2}(x, y), \quad(x, y) \in \Gamma^{N},
$$

where $\Gamma^{D}$ is the boundary segment with Dirichlet boundary condition, $\Gamma^{N}$ the boundary portion with Neumann boundary condition and $\partial \Omega=\Gamma^{D} \cup \Gamma^{N}$ is the whole boundary of computational domain. Besides, $g_{1}(x, y)$ and $g_{2}(x, y)$ are given functions.

\section{$3 \quad$ Numerical methods}

In this paper, we propose the MPS, based on the trigonometric functions, to analyze two-dimensional elliptic equation with constant coefficients. The inhomogeneous term is interpolated by two-dimensional Fourier series and the corresponding particular solutions are derived. In the following subsections, the procedures of the MPS and the derivations of corresponding particular solutions are presented.

\subsection{Method of particular solutions (MPS)}

When the MPS is adopted to analyze boundary value problem, governed by elliptic partial differential equation, Eq. (1), and suitable boundary conditions, Eqs. (2)-(3), the inhomogeneous term of governing equation should be approximated by a series of basis function,

$$
f(x, y) \approx \sum_{j=1}^{N_{m}} c_{j} \phi_{j}(x, y),
$$

where $\left\{c_{j}\right\}_{j=1}^{N_{m}}$ are unknown coefficients to be determined. $N_{m}$ is the number of basis functions for interpolation and also the number of unknown coefficients. $\phi_{j}(x, y)$ is the $j^{\text {th }}$ basis function. The basis functions, $\phi(x, y)$, are the RBFs [8]-[9], the Chebyshev polynomials [19]-[20] and high-order polynomial [21]. In this paper, we use the trigonometric functions to be the basis functions, which will be presented in the next subsection.

Since the inhomogeneous term is interpolated by Eq. (4), the particular solution of the boundary value problem, according to the MPS, can be demonstrated as follows:

$$
u_{p}(x, y)=\sum_{j=1}^{N_{m}} c_{j} \psi_{j}(x, y)
$$


where $u_{p}(x, y)$ is the particular solution of the original problem and it is also the total solution, $u(x, y)$, because it satisfies both the governing equation and boundary conditions in the MPS. $\psi_{j}(x, y)$ is the $j^{t h}$ corresponding basis function. The basis function, $\phi_{j}$, and its corresponding basis function, $\psi_{j}$, should satisfy the original partial differential equation,

$$
\mathcal{L} \psi_{j}(x, y)=\phi_{j}(x, y)
$$

While we used the MPS to analyze the boundary value problem, depicted in Section 2 , two set of nodes are required: interior and boundary nodes. The numbers of interior nodes, boundary nodes along $\Gamma^{D}$, and boundary nodes along $\Gamma^{N}$ are denoted by $n_{i}, n_{b}^{D}$, and $n_{b}^{N}$. Hence, $N_{n}=n_{i}+n_{b}^{D}+n_{b}^{N}$ is the total number of nodes utilized.

Once the solution expressions for particular solution, Eq. (5), and inhomogeneous term, Eq. (4), are obtained, a collocation approach of the MPS can be implemented. To substitute Eq. (5) into Eq. (1) and to enforce the satisfactions of governing equation at every interior node will result in the following system of linear algebraic equations

$$
\sum_{j=1}^{N_{m}} c_{j} \phi_{j}\left(x_{i}, y_{i}\right)=f\left(x_{i}, y_{i}\right), \quad i=1,2,3, \cdots, n_{i} .
$$

In addition, to enforce the satisfactions of boundary conditions along $\Gamma^{D}$ and $\Gamma^{N}$ will yield the following systems,

$$
\sum_{j=1}^{N_{m}} c_{j} \psi_{j}\left(x_{i}, y_{i}\right)=g_{1}\left(x_{i}, y_{i}\right), \quad i=n_{i}+1, n_{i}+2, \cdots, n_{i}+n_{b}^{D},
$$

and

$$
\sum_{j=1}^{N_{m}} c_{j} \frac{\partial \psi_{j}}{\partial n}\left(x_{i}, y_{i}\right)=g_{2}\left(x_{i}, y_{i}\right), \quad i=n_{i}+n_{b}^{D}+1, n_{i}+n_{b}^{D}+2, \cdots, N_{n} .
$$

Via the above approach of collocation, a resultant system of linear algebraic equations is yielded,

$$
\mathrm{Ac}=\mathbf{b}
$$

where $\mathbf{A}$ is the coefficient matrix, $\mathbf{c}=\left[c_{1}, c_{2}, \cdots, c_{N_{m}}\right]^{T}$ is the vector of unknown coefficients, the superscript $T$ denotes the transpose of a vector and $\mathbf{b}$ is formed by inhomogeneous term and boundary conditions. In the resultant system of algebraic equations, Eq. (10), there are $N_{n}$ equations with $N_{m}$ unknown coefficients. In our proposed approach, an over-determined system of algebraic equations $\left(N_{n} \geq N_{m}\right)$ will be formed. The unknown coefficients, c, can be acquired by solving the resultant system of algebraic equations. In addition, the solutions at any spatial position can be calculated by using Eq. (5). The particular solution in Eq. (5) satisfies not only the governing equation, Eq. (7), but also the boundary conditions, Eqs. (8)-(9), so this specific particular solution is absolutely the solution of original boundary value problem. 


\subsection{Derivation of particular solutions for trigonometric func- tions}

The numerical procedures of the MPS are briefly introduced in the previous subsection. From these descriptions, it can be found that the MPS is simple, easy-to-program and truly meshless. In addition to the RBFs, Chebyshev polynomials, and high-order polynomials, we, in this paper, will adopt the trigonometric functions to interpolate the inhomogeneous term of governing equation. The two-dimensional Fourier series is utilized, so Eq. (4) can be shown as,

$$
\begin{aligned}
f(x, y)= & \left(\frac{a_{0}}{2}+\sum_{n=1}^{\infty}\left(a_{n} \cos \left(\frac{n \pi}{L_{x}}\right) x+b_{n} \sin \left(\frac{n \pi}{L_{x}}\right) x\right)\right) \times \\
& \left(\frac{c_{0}}{2}+\sum_{n=1}^{\infty}\left(c_{n} \cos \left(\frac{n \pi}{L_{y}}\right) y+d_{n} \sin \left(\frac{n \pi}{L_{y}}\right) y\right)\right)
\end{aligned}
$$

where $\left\{a_{n}, b_{n}, c_{n}, d_{n}\right\}_{n=0}^{\infty}$ are Fourier coefficients. $L_{x}$ and $L_{y}$ are the x- and y-directional characteristic lengths. Since we use the two-dimensional Fourier series to expand the inhomogeneous term, the rectangle of extended domain, formed by $L_{x}$ and $L_{y}$ as well as displayed in Fig. 1, should be larger than the computational domain according to the assumption of periodic function in Fourier series.

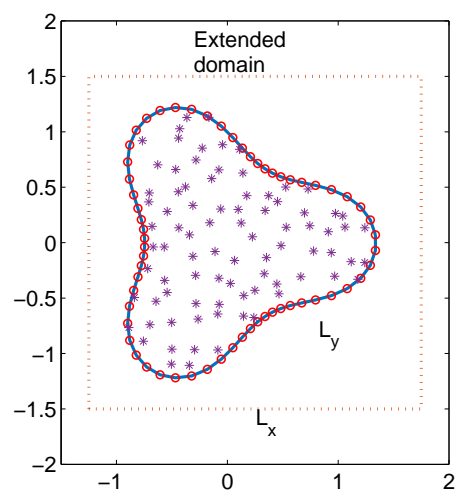

Figure 1: The profile of the extended domain and computational nodes of the MPS.

By expanding and truncating the infinite series of Eq. (11), it can also be written as 
the following form

$$
\begin{array}{r}
f(x, y)=c+\sum_{j=1}^{m}\left(a_{j} \cos \left(h_{j} x\right)+b_{j} \sin \left(h_{j} x\right)+c_{j} \cos \left(k_{j} y\right)+d_{j} \sin \left(k_{j} y\right)\right) \\
+\sum_{i=1}^{m} \sum_{j=1}^{m}\left[\alpha_{i j} \cos \left(h_{i} x\right) \cos \left(k_{j} y\right)+\beta_{i j} \cos \left(h_{i} x\right) \sin \left(k_{j} y\right)\right. \\
\left.+\xi_{i j} \sin \left(h_{i} x\right) \cos \left(k_{j} y\right)+\eta_{i j} \sin \left(h_{i} x\right) \sin \left(k_{j} y\right)\right]
\end{array}
$$

where $h_{j}=j \pi / L_{x}, k_{j}=j \pi / L_{y}$, and $\left\{c, a_{j}, b_{j}, c_{j}, d_{j}, \alpha_{i j}, \beta_{i j}, \xi_{i j}, \eta_{i j}\right\}$ are the unknown coefficients to be determined. $m$ is the adopted order of Fourier series and $N_{m}=4 m^{2}+$ $4 m+1$ is the total number of coefficients. By comparing Eq. (4) with Eq. (12), it is shown that the basis functions, $\phi(x, y)$, include a constant,

$$
\phi(x, y)=1
$$

four sets of trigonometric functions,

$$
\phi(x, y)=\left\{\begin{array}{l}
\cos \left(h_{j} x\right) \\
\sin \left(h_{j} x\right), \\
\cos \left(k_{j} y\right), \\
\sin \left(k_{j} y\right)
\end{array} \quad j=1,2, \cdots m\right.
$$

and four sets of products of two trigonometric functions,

$$
\phi(x, y)=\left\{\begin{array}{l}
\cos \left(h_{x} x\right) \cos \left(k_{j} y\right), \\
\cos \left(h_{i} x\right) \sin \left(k_{j} y\right), \quad i=1,2, \cdots m, \quad j=1,2, \cdots m . \\
\sin \left(h_{i} x\right) \cos \left(k_{j} y\right), \\
\sin \left(h_{i} x\right) \sin \left(k_{j} y\right)
\end{array}\right.
$$

In order to acquire the solution expression of particular solution, Eq. (5), we have to derive the corresponding basis function for every basis function in Eqs. (13)-(15).

\subsubsection{Basis function: constant}

In order to fulfill the relation between basis function and its corresponding basis function of Eq. (6), the corresponding basis function for $\phi(x, y)=1$ can be depicted as

$$
\psi(x, y)=\frac{1}{\gamma}, \quad \text { for } \quad \gamma \neq 0 .
$$




\subsubsection{Basis function: single trigonometric function}

The method of undetermined coefficients for finding particular solution of ordinary differential equation can be adopted to find the particular solution of

$$
\mathcal{L} \psi(x, y)=\cos \left(h_{j} x\right) .
$$

Since the right hand side of the above equation is a cosin function, $\psi$ can be expected to be in the following form

$$
\psi(x, y)=c_{1} \cos \left(h_{j} x\right)+c_{2} \sin \left(h_{j} x\right),
$$

where $c_{1}$ and $c_{2}$ are coefficients to be determined. By substituting (18) into (17), and comparing the coefficients on the both sides, we have

$$
\left[\begin{array}{cc}
-h_{j}^{2}+\gamma & h_{j} \alpha \\
-h_{j} \alpha & -h_{j}^{2}+\gamma
\end{array}\right]\left[\begin{array}{l}
c_{1} \\
c_{2}
\end{array}\right]=\left[\begin{array}{l}
1 \\
0
\end{array}\right] .
$$

Once $c_{1}$ and $c_{2}$ are obtained, the particular solution $\psi(x, y)$ can be determined. For the case of $\mathcal{L} \psi(x, y)=\sin \left(h_{j} x\right)$, the particular solution can be obtained by replacing [ $\left.\begin{array}{ll}1 & 0\end{array}\right]^{T}$ in the right hand side of (19) with $\left[\begin{array}{ll}0 & 1\end{array}\right]^{T}$.

Similarly, for the case of $\mathcal{L} \psi(x, y)=\cos \left(k_{j} y\right)$, we can arrive the following system of equations

$$
\left[\begin{array}{cc}
-k_{j}^{2}+\gamma & k_{j} \beta \\
-k_{j} \beta & -k_{j}^{2}+\gamma
\end{array}\right]\left[\begin{array}{l}
c_{1} \\
c_{2}
\end{array}\right]=\left[\begin{array}{l}
1 \\
0
\end{array}\right] .
$$

For $\mathcal{L} \psi(x, y)=\sin \left(k_{j} y\right)$, the particular solution $\psi$ can be obtained by replacing [ $\left.\begin{array}{ll}1 & 0\end{array}\right]^{T}$ in the right hand side of (20) with [ $\left.0 \begin{array}{ll}0 & 1\end{array}\right]^{T}$.

\subsubsection{Basis function: product of two trigonometric functions}

To obtain the particular solution $\psi$ for the cases of product terms as shown in (15), we again apply the method of undetermined coefficients as shown in the previous subsection. For the case

$$
\mathcal{L} \psi(x, y)=\cos \left(h_{j} x\right) \cos \left(k_{j} y\right),
$$

we can start by assuming

$$
\begin{aligned}
\psi(x, y)= & d_{1} \cos \left(h_{j} x\right) \cos \left(k_{j} y\right)+d_{2} \cos \left(h_{j} x\right) \sin \left(k_{j} y\right) \\
& +d_{3} \sin \left(h_{j} x\right) \cos \left(k_{j} y\right)+d_{4} \sin \left(h_{j} x\right) \sin \left(k_{j} y\right),
\end{aligned}
$$

where $d_{i}, i=1,2,3,4$, are coefficients to be determined. Substituting (22) into (21), we can obtain the following system of equations

$$
\left[\begin{array}{cccc}
-h_{i}^{2}-k_{j}^{2}+\gamma & k_{j} \beta & h_{i} \alpha & 0 \\
-k_{j} \beta & -h_{i}^{2}-k_{j}^{2}+\gamma & 0 & h_{i} \alpha \\
-h_{i} \alpha & 0 & -h_{i}^{2}-k_{j}^{2}+\gamma & k_{j} \beta \\
0 & -h_{i} \alpha & -k_{j} \beta & -h_{i}^{2}-k_{j}^{2}+\gamma
\end{array}\right]\left[\begin{array}{l}
d_{1} \\
d_{2} \\
d_{3} \\
d_{4}
\end{array}\right]=\left[\begin{array}{l}
1 \\
0 \\
0 \\
0
\end{array}\right]
$$


$1 \leq i, j \leq m$. The other three cases in (15) can be obtained by replacing the right hand side of (23) with $\left[\begin{array}{llll}0 & 1 & 0 & 0\end{array}\right]^{T},\left[\begin{array}{llll}0 & 0 & 1 & 0\end{array}\right]^{T}$, and $\left[\begin{array}{llll}0 & 0 & 0 & 1\end{array}\right]^{T}$ respectively.

\section{Numerical results and comparisons}

In this section, five numerical examples are provided to examine the accuracy of the proposed MPS and test the influence of factors in the proposed method. The following equations are used to calculate the maximum absolute error (MAE) and maximum relative error (MRE) of numerical solutions,

$$
\begin{aligned}
& M A E=\max _{1 \leq j \leq N_{t}}\left|u_{j}-\hat{u}_{j}\right|, \\
& M R E=\max _{1 \leq j \leq N_{t}} \frac{\left|u_{j}-\hat{u}_{j}\right|}{\left|u_{j}\right|},
\end{aligned}
$$

where $N_{t}$ is the number of test nodes, and the test nodes, which are different from the collocation nodes, are randomly distributed inside the computational domain. $u_{j}$ and $\hat{u}_{j}$ are the analytical solution and numerical result at the $j^{\text {th }}$ nodes.

Example 1 Consider the following boundary value problem

$$
\begin{aligned}
\left(\nabla^{2}-2 \frac{\partial}{\partial x}+\frac{\partial}{\partial y}+3\right) u(x, y) & =f(x, y), \quad(x, y) \in \Omega, \\
u(x, y) & =g(x, y), \quad(x, y) \in \partial \Omega
\end{aligned}
$$

where $f$ and $g$ are given based on the following exact solution

$$
u(x, y)=x^{2}+y^{2}+x+y+1 .
$$

The profile of $\Omega$ and its boundary $\partial \Omega$ are shown in Figure 2(a) and the parametric equation of $\partial \Omega$ is given as follows

$$
\partial \Omega=\{(x, y): x=r \cos \theta, y=r \sin \theta, 0 \leq \theta \leq 2 \pi\}
$$

where

$$
r=\left(\cos (2 \theta)+\sqrt{1.1-\sin ^{2}(2 \theta)}\right)^{1 / 3} .
$$

The profile of exact solution is shown in Fig. 2(b).

For the Dirichlet boundary condition, we choose $L_{x}=2.6, L_{y}=1.1, N_{n}=1872, n_{i}=$ $1672, N_{m}=625, m=12$, and $N_{t}=3100$. In this simulation we obtain $M A E=9.302 \times$ $10^{-8}$ and $M R E=8.813 \times 10^{-8}$ which is highly accurate. The profiles of numerical errors for various $N_{n}$ and $m$ are shown in Fig. 3(a). In Fig. 3(b), we observe the 


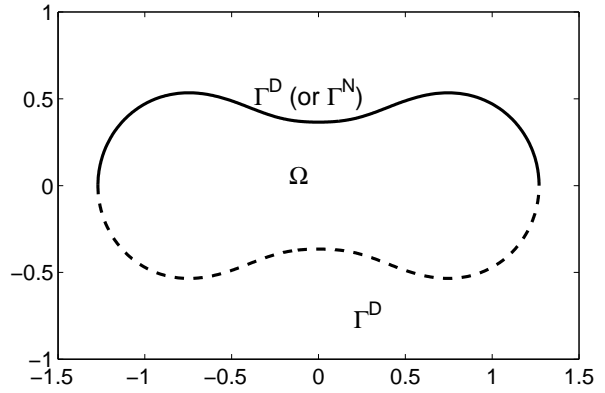

(a) Computational domain

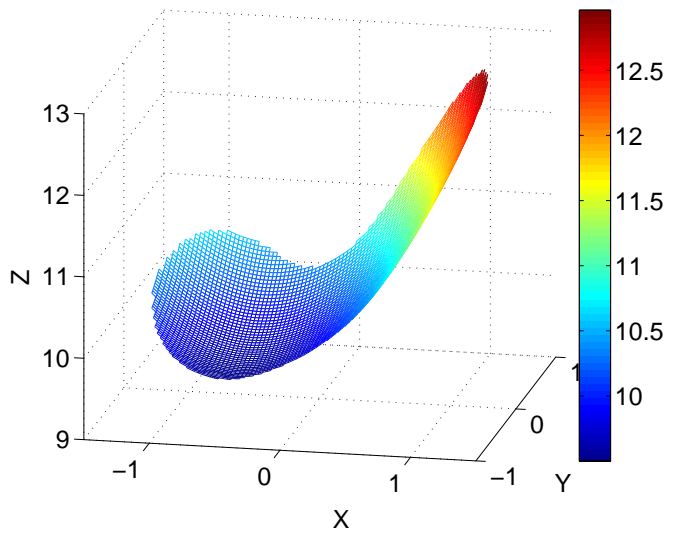

(b) Exact solution

Figure 2: Example 1: The profiles of (a) the computational domain and (b) exact solution.

increase of the number of order $m$ will dramatically improve the accuracy. Moreover, five different sets of characteristic lengths are used and the numerical errors are depicted in Table 1. In this table, it can be found that the results are highly accurate for these five sets of characteristic lengths. It is also worth noticing that the better solutions can be obtained if the characteristic lengths of the extended domain are slightly larger than the computational domain.

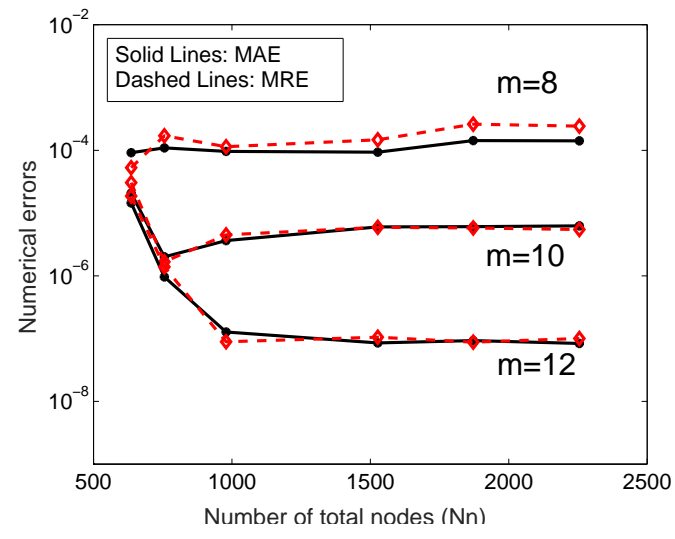

(a)

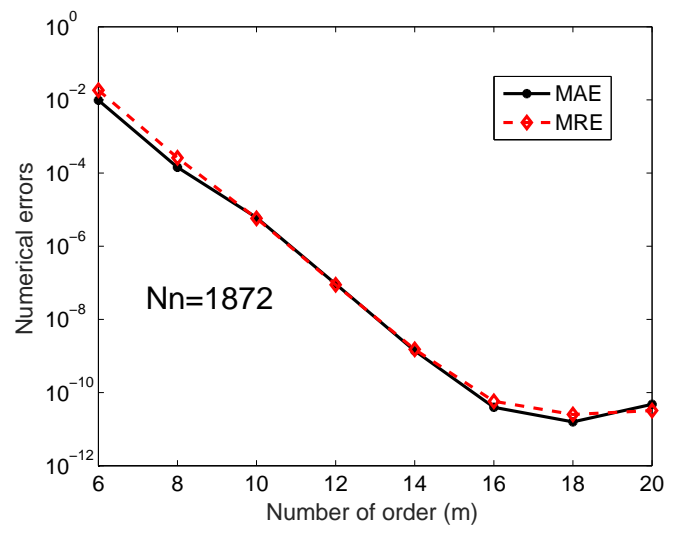

(b)

Figure 3: Example 1: Numerical errors for Dirichlet boundary condition using (a) different numbers of total nodes $\left(N_{n}\right)$ and (b) different numbers of order $(m)$.

Next, we consider the mixed boundary conditions where the Neumann and Dirichlet boundary condition are imposed along the upper and lower half boundary segments respectively. The parameters are identical to the above test. The MAE is $3.524 \times 10^{-7}$ and the MRE is $2.641 \times 10^{-7}$ for the second test, which validate the highly-accurate 


\begin{tabular}{cccccc}
\hline$L_{x}$ & 2.6 & 2.8 & 3.2 & 5 & 20 \\
\hline$L_{y}$ & 1.1 & 1.2 & 1.4 & 2 & 10 \\
\hline MAE & $9.3 \times 10^{-8}$ & $1.1 \times 10^{-8}$ & $3.5 \times 10^{-10}$ & $5.6 \times 10^{-12}$ & $2.2 \times 10^{-11}$ \\
\hline MRE & $8.8 \times 10^{-8}$ & $1.3 \times 10^{-8}$ & $3.9 \times 10^{-10}$ & $8.4 \times 10^{-12}$ & $3.2 \times 10^{-11}$ \\
\hline
\end{tabular}

Table 1: Example 1: Numerical errors using different characteristic lengths of extended domains for Dirichlet boundary condition. $\left(N_{n}=1872, m=12\right)$

results using the proposed MPS. Besides, different numbers of total nodes and different orders are utilized in this test, and the profiles of numerical errors are demonstrated in Fig. 4. In both figures, the numerical errors are very small and the errors decrease rapidly by using either more nodes $N_{n}$ or larger order $m$. In addition, the numerical errors by using different characteristic lengths are presented in Table 2 . The numerical accuracy is excellent using these five lengths. The optimal errors can be acquired when the characteristic lengths are slightly larger than the size of computational domain.

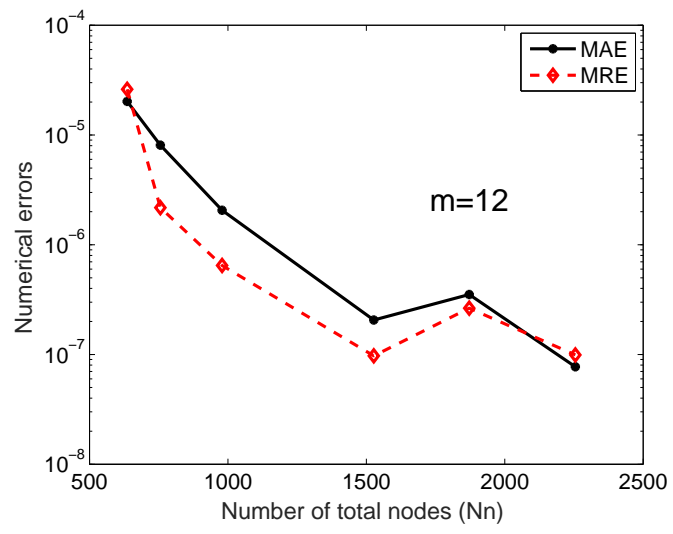

(a)

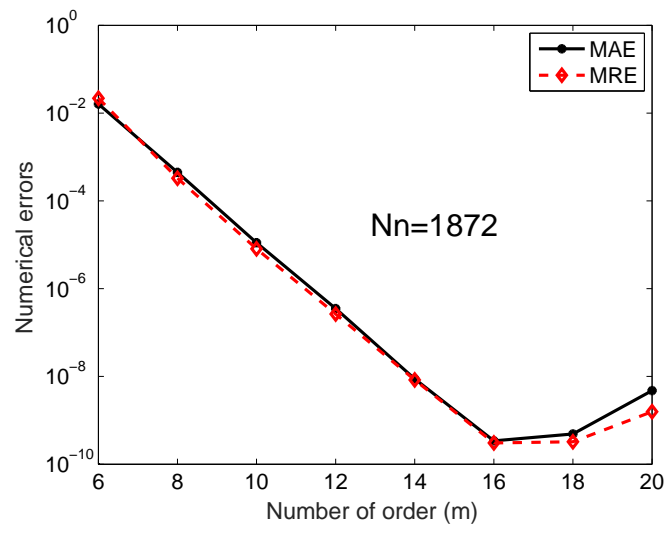

(b)

Figure 4: Example 1: Numerical errors for mixed boundary conditions using (a) different numbers of total nodes $\left(N_{n}\right)$ and (b) different numbers of order $(m)$.

\begin{tabular}{cccccc}
\hline$L_{x}$ & 2.6 & 2.8 & 3.2 & 5 & 20 \\
\hline$L_{y}$ & 1.1 & 1.2 & 1.4 & 2 & 10 \\
\hline MAE & $3.5 \times 10^{-8}$ & $2.9 \times 10^{-8}$ & $7.9 \times 10^{-10}$ & $1.7 \times 10^{-12}$ & $8.4 \times 10^{-11}$ \\
\hline MRE & $2.6 \times 10^{-8}$ & $2.3 \times 10^{-8}$ & $5.7 \times 10^{-10}$ & $1.7 \times 10^{-12}$ & $1.6 \times 10^{-11}$ \\
\hline
\end{tabular}

Table 2: Example 1: Numerical errors using different characteristic lengths of extended domains for mixed boundary condition. $\left(N_{n}=1872, m=12\right)$

In Figure 5, we show the numerical errors using the Kansa method with MQ with $N_{n}=$ 1872. Note that the uncertainty of the shape parameter is still an outstanding research 
problem. The best results we can obtain are E-07 for Dirichlet boundary condition and E06 for the mixed boundary condition. In comparison, our proposed approach is far more superior than using the RBF collocation method. From the numerical results obtained in these two tests, it can be verified that the proposed trigonometric functions-based MPS can achieve excellent accuracy without the headache of the free-parameters of the RBFs. The numerical errors can be improved by increasing either the number of total nodes or the order of expansion and choosing the optimal characteristic lengths.

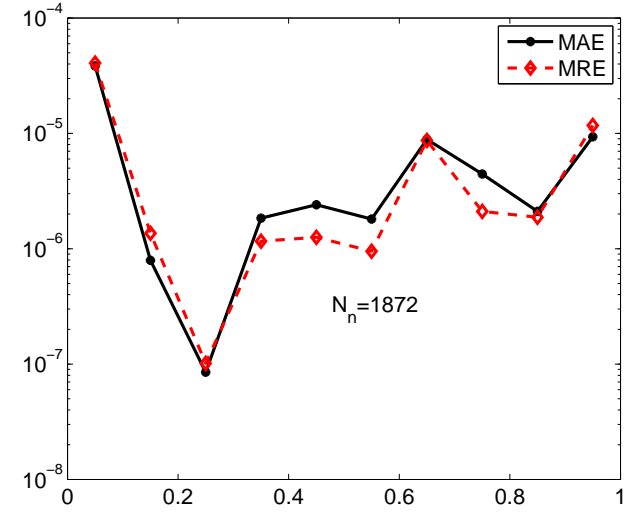

(a) Dirichlet boundary condition

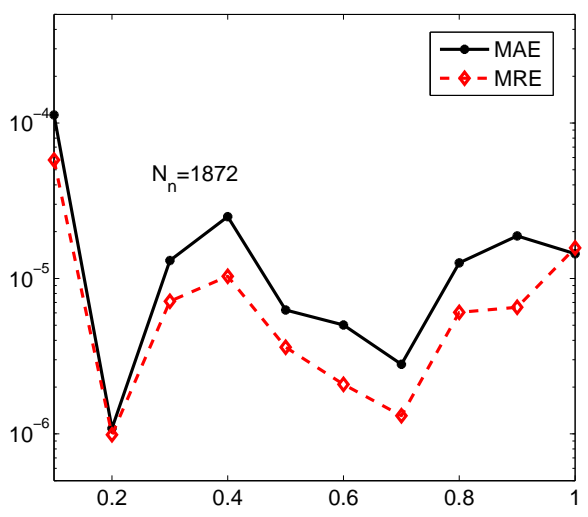

(b) Mixed boundary condition

Figure 5: Example 1: Numerical errors using RBF-MQ with $N_{n}=1827$ (a) for Dirichlet boundary condition (b) mixed boundary condition.

Example 2 In this example, we consider a double connected domain as shown in Fig. $6(\mathrm{a})$. The inner boundary is formed by a circle with radius 0.5 and centered at the origin. The outer boundary is described by the following parameter equation,

$$
\partial \Omega=\left\{(x, y): x=\left(1+\cos ^{2}(4 \theta)\right) \cos \theta, y=\left(1+\cos ^{2}(4 \theta)\right) \sin \theta, 0 \leq \theta \leq 2 \pi\right\} .
$$

We consider the following boundary value problem

$$
\begin{array}{rlrl}
\left(\nabla^{2}+\frac{\partial}{\partial x}-2 \frac{\partial}{\partial y}+1\right) u(x, y) & =f(x, y), & (x, y) & \in \Omega, \\
u(x, y) & =g(x, y), \quad(x, y) & \in \Gamma^{D}, \\
\frac{\partial u}{\partial n}(x, y) & =h(x, y), & (x, y) & \in \Gamma^{N},
\end{array}
$$

where $\Gamma^{D}$ is outer boundary and $\Gamma^{N}$ the inner boundary. $f, g$ and $h$ are given based on the following exact solution

$$
u(x, y)=e^{x} \cos y+e^{y} \sin x+x+y+10 .
$$




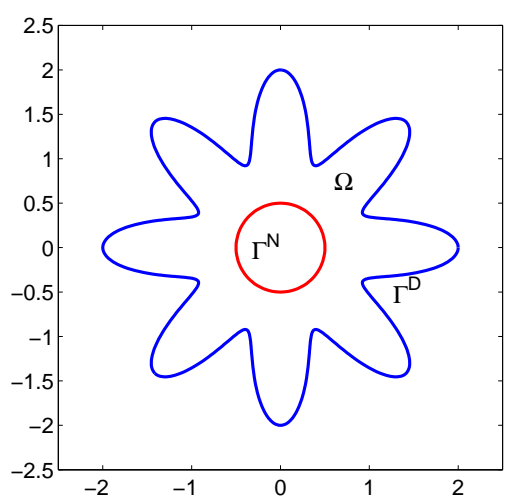

(a) Computational domain

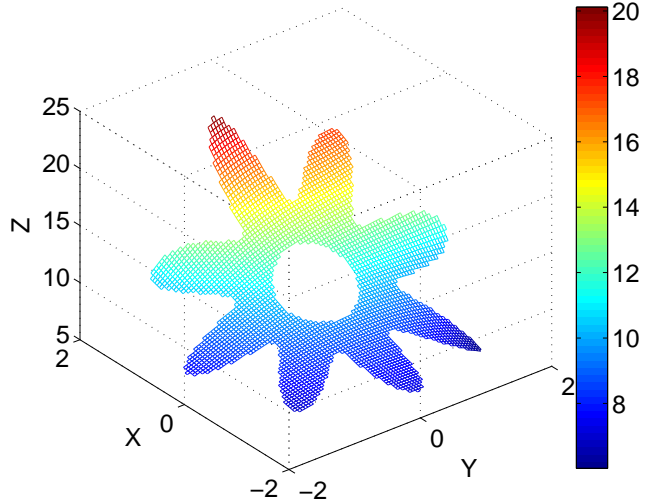

(b) Exact solution

Figure 6: Example 2: The profiles of (a) the computational domain (b) exact solution.

The profile of the exact solution is shown in Fig. 6(b).

In the numerical implementation, we choose $L_{x}=L_{y}=4, N_{n}=1780, n_{b}^{D}=300, n_{b}^{N}=$ $30, N_{m}=625, m=12$, and $N_{t}=3025$. In this calculation, the MAE is $1.959 \times 10^{-7}$ and the MRE is $2.666 \times 10^{-7}$, which show the high accuracy of the proposed MPS. Also, the numerical errors are shown in Fig. 7 using various numbers of $N_{n}$ and $m$. From Fig. 7 (a), the numerical errors become stable for $N_{n}>1153$. In Fig. 7(b), the numerical errors diminish rapidly with increasing $m$ and become extremely small using few order of series expansion. Moreover, we used five different characteristic lengths to solve this example and the numerical errors are shown in Table 3. Again, we observe the excellent accuracy.

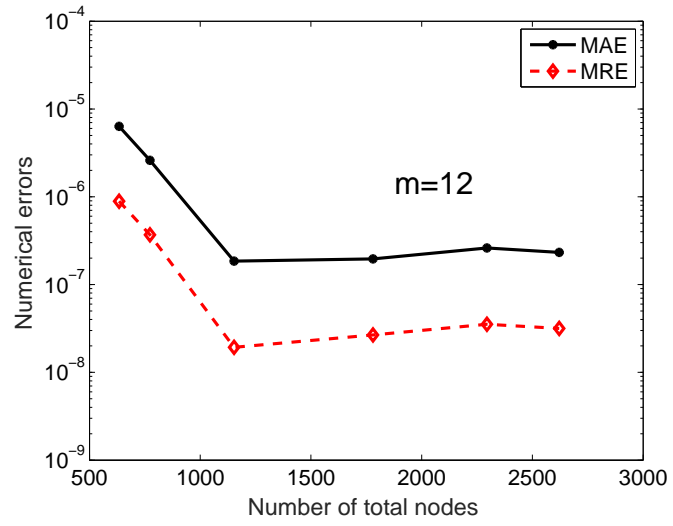

(a)

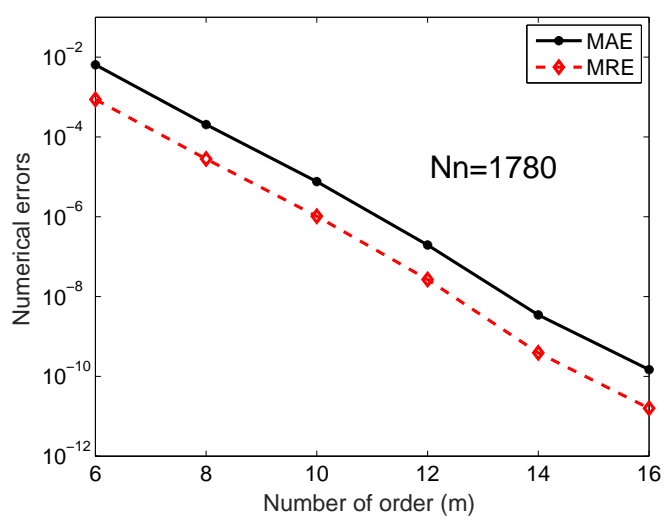

(b)

Figure 7: Example 2: Profiles of numerical errors by using (a) different numbers of total nodes $\left(N_{n}\right)$ and $(\mathrm{b})$ different numbers of order $(m)$ in expansion. 


\begin{tabular}{cccccc}
\hline$L_{x}=L_{y}$ & 4 & 4.5 & 5 & 7 & 10 \\
\hline MAE & $2.0 \times 10^{-7}$ & $1.1 \times 10^{-8}$ & $1.1 \times 10^{-9}$ & $4.4 \times 10^{-10}$ & $6.0 \times 10^{-11}$ \\
\hline MRE & $2.7 \times 10^{-8}$ & $1.3 \times 10^{-9}$ & $1.5 \times 10^{-10}$ & $5.1 \times 10^{-11}$ & $6.0 \times 10^{-12}$ \\
\hline
\end{tabular}

Table 3: Example 2: Numerical errors using different characteristic lengths of extended domains. $\left(N_{n}=1780, m=12\right)$

Example 3 In this example, we consider the following modified Helmholtz equation

$$
\begin{aligned}
\left(\nabla^{2}-100\right) u(x, y) & =f(x, y), & (x, y) \in \Omega, \\
u(x, y) & =g(x, y), & (x, y) \in \partial \Omega,
\end{aligned}
$$

where $f$ and $g$ are given functions based on the following exact solution

$$
u(x, y)=\sin x \cosh y-\cos x \sinh y+8 .
$$

The parametric equation of the boundary $\partial \Omega$ is given as follows:

$$
\partial \Omega=\{(x, y): x=r(\theta) \cos (\sigma(\theta)), y=r(\theta) \sin (\sigma(\theta)), 0 \leq \theta \leq 2 \pi\},
$$

where

$$
r(\theta)=2+\frac{1}{2} \sin (6 \theta), \quad \sigma(\theta)=\theta+\frac{1}{2} \sin (6 \theta) .
$$

The profiles of the computational domain and exact solution are shown in Fig. 8.

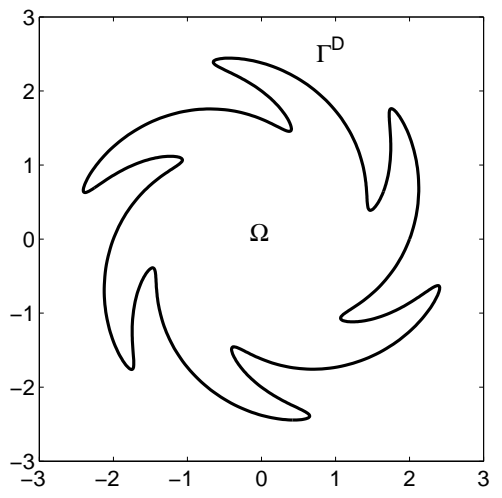

(a) Computational Doamin

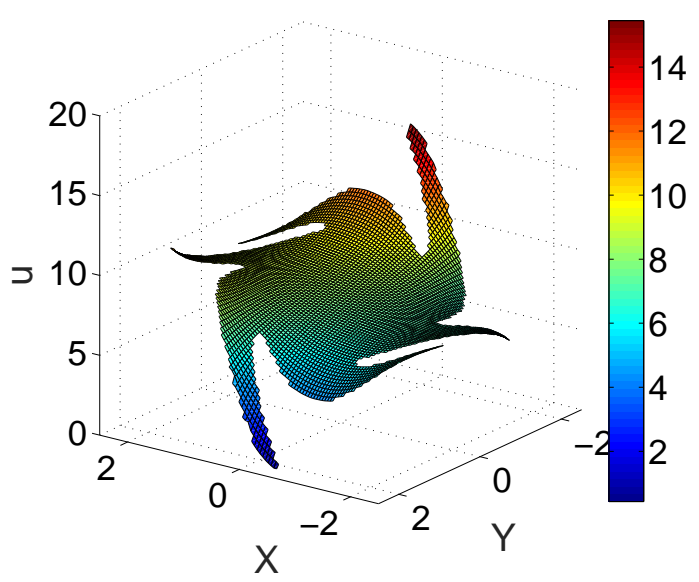

(b) Exact solution

Figure 8: Example 3: The profiles of the (a) computational domain (b) exact solution.

In this example, the following parameters are used: $L_{x}=L_{y}=5.5, N_{m}=625, m=$ $12, N_{n}=2253, n_{b}^{D}=500$ and $N_{t}=3589$. The numerical errors are MAE $=2.187 \times 10^{-8}$ 
and $\mathrm{MRE}=3.511 \times 10^{-8}$. The numerical errors for various $N_{n}$ and $m$ are shown in Fig. 9. In Fig. 9(a), the numerical errors are quite small and stable. Besides, in Fig. 9(b), the numerical errors can be diminished by adopting a larger order of series expansion. Table 4 shows the numerical errors by using five different characteristic lengths of extended domain. The smaller errors is observed when the characteristic length is larger than the actual size of computational domain. These results is consistent with the results obtained in the previous examples.

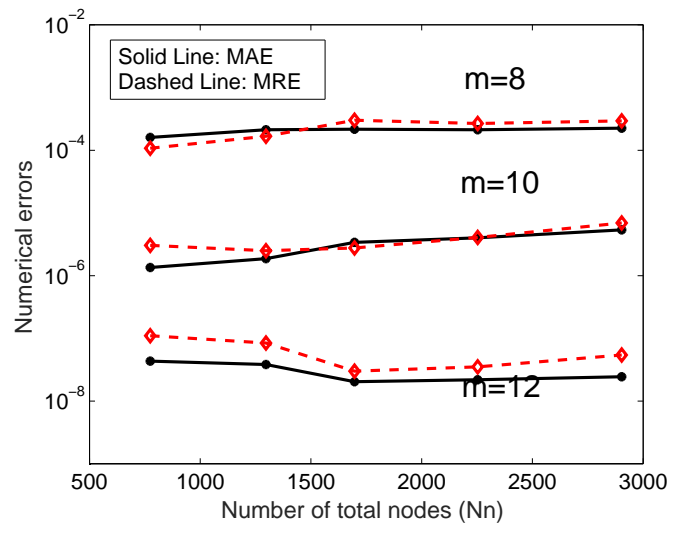

(a)

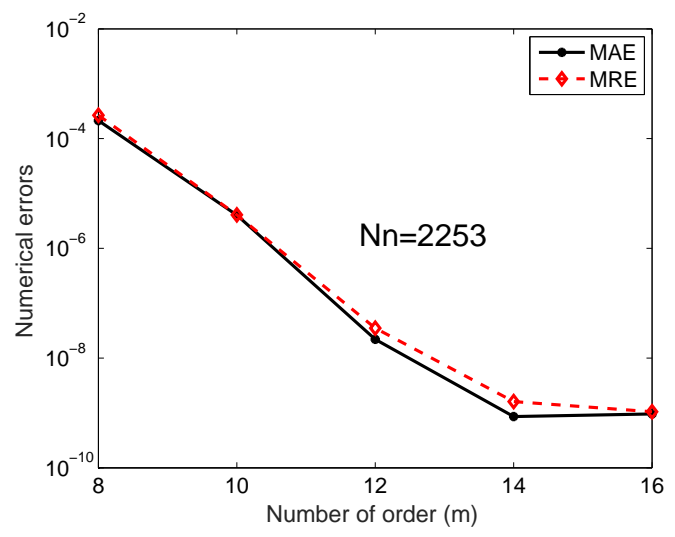

(b)

Figure 9: Example 3: Profiles of numerical errors by using (a) different numbers of total nodes $\left(N_{n}\right)$ and $(\mathrm{b})$ different numbers of order $(m)$ in expansion.

\begin{tabular}{cccccc}
\hline$L_{x}=L_{y}$ & 5.5 & 6 & 7 & 8 & 10 \\
\hline MAE & $2.0 \times 10^{-8}$ & $6.0 \times 10^{-9}$ & $1.5 \times 10^{-9}$ & $3.4 \times 10^{-9}$ & $6.3 \times 10^{-9}$ \\
\hline MRE & $3.5 \times 10^{-8}$ & $1.8 \times 10^{-8}$ & $7.0 \times 10^{-9}$ & $8.1 \times 10^{-9}$ & $9.3 \times 10^{-9}$ \\
\hline
\end{tabular}

Table 4: Example 3: Numerical errors using different characteristic lengths of extended domains. $\left(N_{n}=2253, m=12\right)$

Example 4 Consider the following boundary value problem with large convective coefficients

$$
\begin{array}{rlrl}
\left(\nabla^{2}+100 \frac{\partial}{\partial x}+100 \frac{\partial}{\partial y}+1\right) u(x, y) & =f(x, y), \quad(x, y) & \in \Omega, \\
u(x, y) & =g(x, y), \quad(x, y) & \in \Gamma^{D}, \\
\frac{\partial u}{\partial n}(x, y) & =h(x, y), \quad(x, y) \in \Gamma^{N},
\end{array}
$$

where $f, g$ and $h$ are given based on the following exact solution

$$
u(x, y)=\sin (7.5 x) \cos (10.7 y)+2 .
$$


Fig. 10(a) show the profile of computational domain $\Omega$ and its boundary $\Gamma^{D}$ which is along two straight boundary segments, and $\Gamma^{N}$ along the arc. The profile of exact solution is shown in Fig. 10(b).

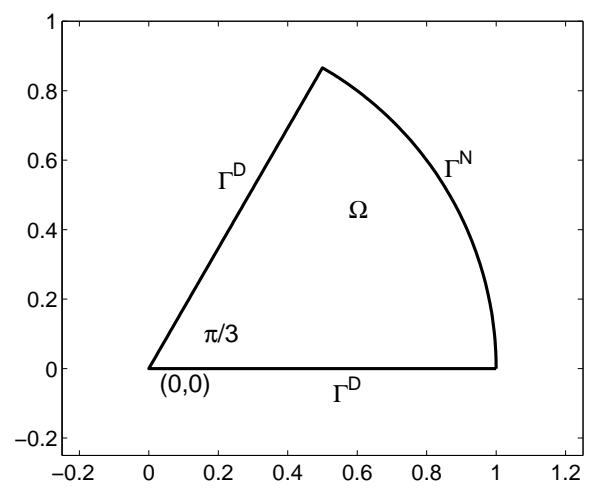

(a) Computational domain

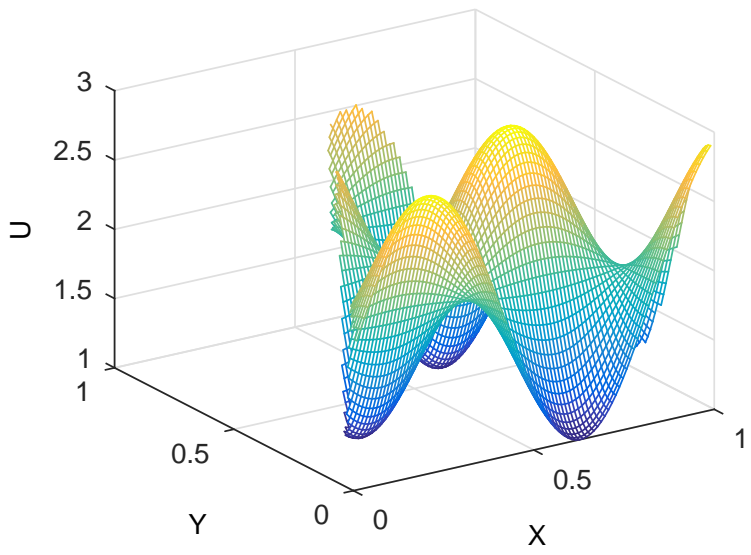

(b) Exact solution

Figure 10: Example 4: The profile of the computational domain.

In the numerical implementation, we set $L_{x}=L_{y}=1.5, N_{m}=289, m=8, N_{n}=$ $1268, n_{b}^{D}=140, n_{i}=1058$, and $N_{t}=4240$. The numerical errors are MAE $=9.778 \times 10^{-8}$ and MRE $=4.743 \times 10^{-8}$. The numerical errors for various $N_{n}$ and $m$ are illustrated in Fig. 11 where excellent accuracy has been achieved for various $N_{n}$ and $m$. As shown in Table 5, similar results as before have been obtained for various characteristic lengths.

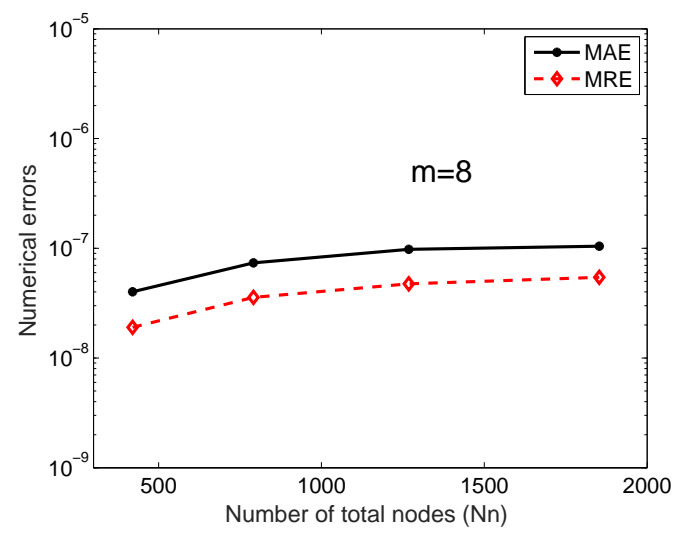

(a)

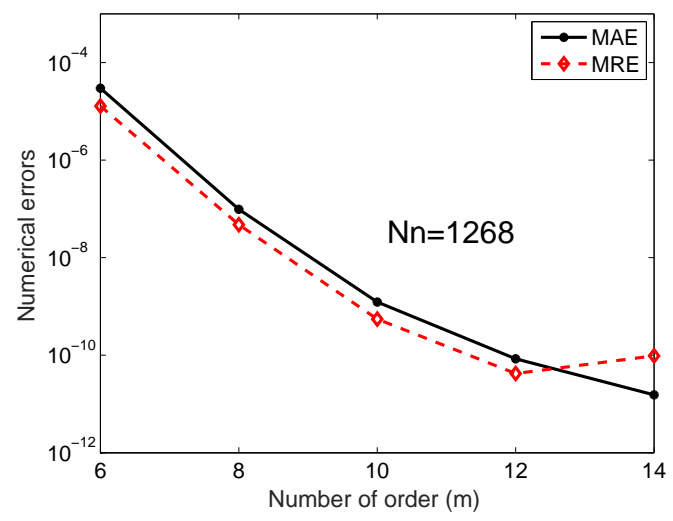

(b)

Figure 11: Example 4: Profiles of numerical errors by using (a) different numbers of total nodes $\left(N_{n}\right)$ and $(\mathrm{b})$ different numbers of order $(m)$ in expansion. 


\begin{tabular}{cccccc}
\hline$L_{z}=L_{y}$ & 1 & 1.5 & 2 & 2.5 & 3 \\
\hline MAE & $1.0 \times 10^{-4}$ & $9.8 \times 10^{-9}$ & $4.3 \times 10^{-9}$ & $1.2 \times 10^{-7}$ & $1.1 \times 10^{-5}$ \\
\hline MRE & $7.8 \times 10^{-5}$ & $4.7 \times 10^{-8}$ & $1.7 \times 10^{-9}$ & $5.9 \times 10^{-8}$ & $7.3 \times 10^{-6}$ \\
\hline
\end{tabular}

Table 5: Example 4: Numerical errors using different characteristic lengths of extended domains. $\left(N_{n}=1268, m=8\right)$

Example 5 In this example, we consider the following well-known Runge problem in a square domain $[-1,1]^{2}$ :

$$
\begin{array}{rlrl}
\nabla^{2} u(x, y) & =f(x, y), & (x, y) \in \Omega \\
u(x, y) & =g(x, y), & & (x, y) \in \partial \Omega
\end{array}
$$

where $f$ and $g$ are given according to the analytical solution

$$
u(x, y)=\frac{1}{1+25\left(x^{2}+y^{2}\right)} .
$$

The profile of the exact solution is shown in Figure 12(a).

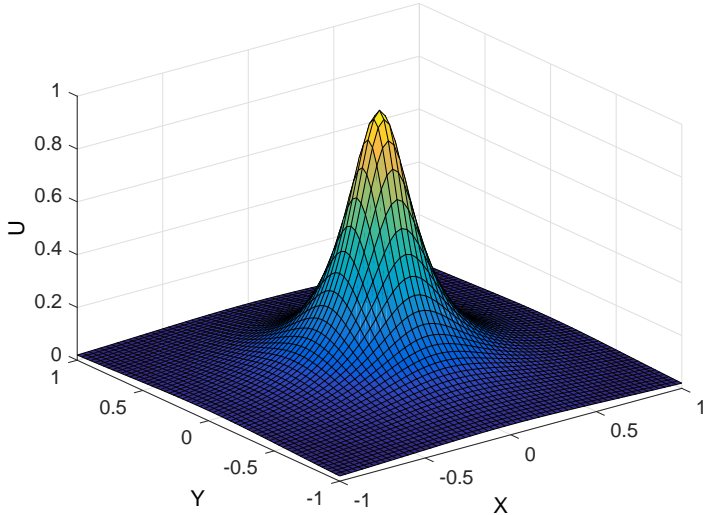

(a) Exact solution

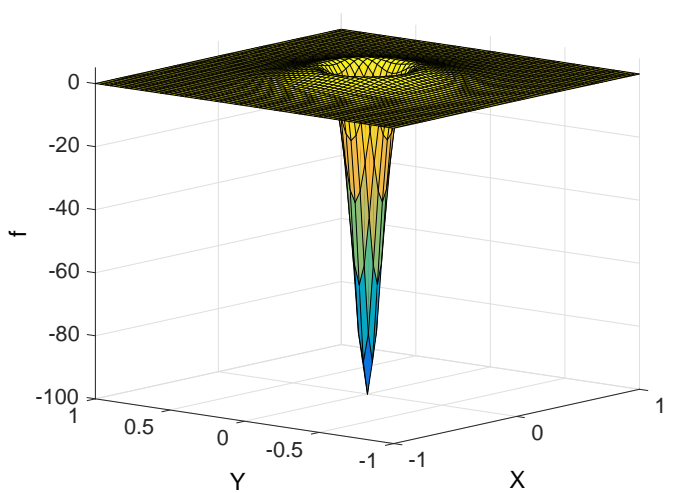

(b) Forcing term $f(x, y)$

Figure 12: Example 5: The profile of (a) the exact solution (b) forcing term $f(x, y)$.

The Runge phenomenon, which means inaccurate oscillations near boundary, usually appears in the numerical solution of Runge function by collocation approaches, and is known as a challenging problem. The outlook of exact solution is deceiving. In fact, the forcing term $f(x, y)$ as shown in Figure 12(b) has a sharp spike at the origin which makes the problem much harder to handle. 
Since all coefficients of governing equation, $\alpha, \beta$ and $\gamma$, are equal to zero, the corresponding basis function for $\phi(x, y)=1$ is depicted as follows.

$$
\psi(x, y)=\frac{x^{2}}{2}
$$

In the numerical implementation, we choose $L_{x}=L_{y}=3, n_{b}^{D}=480, N_{m}=10201, m=50$ and $N_{t}=3595, N_{n}=14880$.

It can be noticed that the numbers of nodes and order, utilized in this example, is much larger than the previous four examples due to the difficulty of the Runge phenomenon. The numerical results show that MAE is $3.29 \times 10^{-4}$ while MRE is $1.54 \times 10^{-2}$. Although these two numerical errors are not as good as those in the previous examples, the numerical errors are acceptable. Also, we adopted different numbers of total nodes and different orders of series expansion to analyze this Runge problem. The profiles of numerical errors are revealed in Fig. 13. Numerical errors are very similar to each other for $m=50$ using various $N_{n}$. The tendency of numerical errors in Fig. 13(a) implies that the numerical accuracy cannot be improved by only increasing the number of nodes when a fixed order of series expansion $(m=50)$ is used. Therefore, the numerical errors by using different orders of expansion and a fixed number of total nodes are demonstrated in Fig. 13(b). It is evident that the numerical errors can be rapidly decreased by increasing the order of approximation. In addition, we test the influence of different characteristic lengths on numerical accuracy. The numerical results and comparisons are shown in Table 6. We adopted five different characteristic lengths in this table and all tests can acquire acceptable results. Similar with previous examples, the optimal numerical results can be acquired while the characteristic length is slightly larger than the size of computational domain.

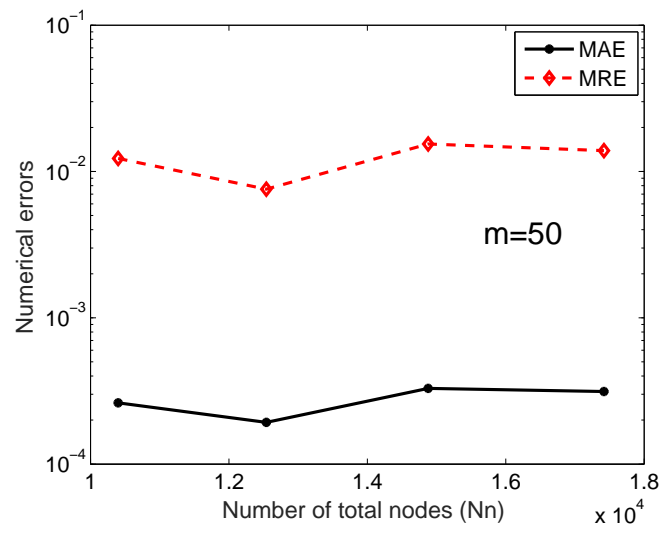

(a)

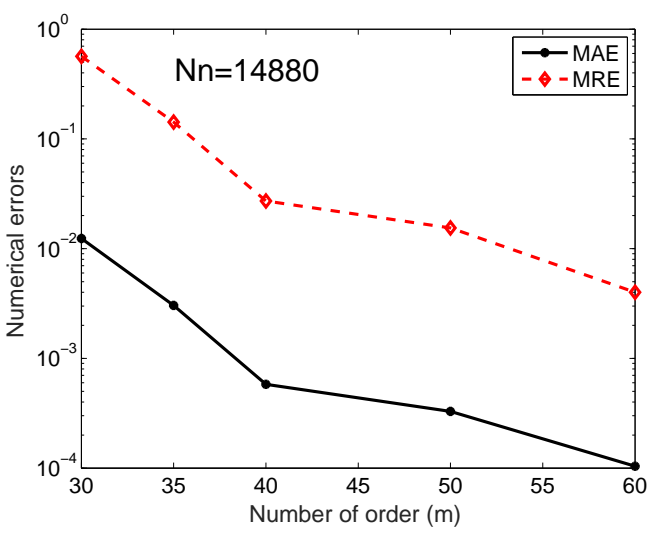

(b)

Figure 13: Example 5: Numerical errors using (a) different numbers of total nodes $\left(N_{n}\right)$ and $(b)$ different numbers of order $(m)$ in series expansion. 


\begin{tabular}{cccccc}
\hline$L_{x}=L_{y}$ & 2.5 & 3 & 3.5 & 4 & 5 \\
\hline MAE & $7.1 \times 10^{-5}$ & $3.3 \times 10^{-4}$ & $2.6 \times 10^{-3}$ & $2.9 \times 10^{-3}$ & $4.9 \times 10^{-3}$ \\
MRE & $2.7 \times 10^{-3}$ & $1.5 \times 10^{-2}$ & $1.2 \times 10^{-1}$ & $1.2 \times 10^{-1}$ & $2.3 \times 10^{-1}$ \\
\hline
\end{tabular}

Table 6: Example 5: Numerical errors using different characteristic lengths of extended domains. $\left(N_{n}=14880, m=50\right)$

\section{Conclusions}

The MPS, based on trigonometric functions, is proposed in this paper to accurately analyze two-dimensional elliptic equations. The inhomogeneous term is approximated by two-dimensional Fourier series and the corresponding basis functions are derived in this paper. In comparing with the MPS used RBFs, the Chebyshev polynomials and highorder polynomials, the proposed trigonometric functions-based MPS is free from choosing the free parameter in RBFs, locating the collocation points at some special positions and dealing with highly ill-conditioning matrices. Five numerical examples are provided in this paper to verify the merits of the proposed method. These examples includes problems with mixed boundary conditions, problem in doubly-connected domain, modified Helmholtz equation, problem with large convective coefficients and Poisson equation (Runge problem) with $\alpha=\beta=\gamma=0$. Furthermore, the influence of number of total nodes, the order of Fourier series and the characteristic lengths of extended domain on accuracy are systematically investigated in these examples. The numerical results are compared very well with analytical solutions in all of these examples. From the comparisons in these examples, it can be deduced that the numerical errors can be decreased if more interpolation nodes and large order in expansion are adopted. Also, an extended domain, which is slightly larger than the computational domain, is suggested to acquire more accurate results.

After the successful validations of the proposed meshless method in this paper, it is worth to study some applications of the proposed trigonometric functions-based MPS in the future. Recently, we are working on the extensions of the proposed meshless approach to three-dimensional elliptic problems, inverse Cauchy problems, and fluid flow-related problems. Furthermore, it is also interesting to figure out the localized scheme of the proposed MPS.

\section{Acknowledgements}

The first author acknowledges the partial support of the National Natural Science Foundation of China (Project No. 11401422). The corresponding author acknowledges the support of the National Natural Science Foundation of China (Project No. 11201288). 


\section{References}

[1] J.H. Ferziger and M. Peric, Computational Methods for Fluid Dynamics, 3rd rev. ed., Springer, Berlin, 2002.

[2] R.W. Lewis, P. Nithiaramu and K.N. Seetharamu, Fundamentals of the Finite Element Method for Heat and Fluid Flow, John Wiley \& Sons Ltd., Chichester, West Sussex, 2004.

[3] A.H.-D. Cheng and D.T. Cheng, Heritage and early history of the boundary element method, Engineering Analysis with Boundary Elements, 29, 268-302, 2005.

[4] G.R. Liu, Meshfree Methods: Moving beyond the Finite Element Method, 2nd ed., CRC Press, Boca Raton, 2010.

[5] R. Mathon and R.L. Johnston, The approximate solution of elliptic boundary-value problems by fundamental solutions, SIAM Journal on Numerical Analysis, 14(4), 638-650, 1977.

[6] G. Fairweather and A. Karageorghis, The method of fundamental solutions for elliptic boundary value problems, Advances in Computational Mathematics, 9, 69-95, 1998.

[7] M.A. Golberg, The method of fundamental solutions for Poisson's equation, Engineering Analysis with Boundary Elements, 16, 205-213, 1995.

[8] C.S. Chen, C.M. Fan and P.H. Wen, The method of approximate particular solutions for solving certain partial differential equations, Numerical Methods for Partial Differential Equations, 28, 506-522, 2012.

[9] C.S. Chen, C.M. Fan and P.H. Wen, The method of approximate particular solutions for solving elliptic problems with variable coefficients, International Journal of Computational Methods, 8(3), 545-559, 2011.

[10] H.F. Chan, C.M. Fan and C.W. Kuo, Generalized finite difference method for solving two-dimensional non-linear obstacle problems, Engineering Analysis with Boundary Elements, 37, 1189-1196, 2013.

[11] P.C. Guan and C.Y. Sun, The isoparametric reproducing kernel particle method for nonlinear deformation of plates, Engineering Analysis with Boundary Elements, 42, 67-76, 2014.

[12] C.S. Chen, T.O. Kwok and L. Ling, Adaptive method of particular solution for solving 3D inhomogeneous elliptic equations, International Journal of Computational Methods, 7(3), 499-511, 2010. 
[13] C.A. Bustamante, H. Power, W.F. Florez and C.Y. Hang, The global approximate particular solution meshless method for two-dimensional linear elasticity problems, International Journal of Computer Mathematics, 90(5), 978-993, 2012.

[14] C.A. Bustamante, H. Power and W.F. Florez, A global meshless collocation particular solution method for solving the two-dimensional Navier-Stokes system of equations, Computers and Mathematics with Applications, 65(12), 1939-1955, 2013.

[15] T. Jiang, M. Li and C.S. Chen, The method of particular solutions for solving inverse problems of a nonhomogeneous convection-diffusion equation with variable coefficients, Numerical Heat Transfer, Part A, 64, 338-352, 2012.

[16] Z.J. Fu, W. Chen and L. Ling, Method of approximate particular solutions for constant- and variable-order fractional diffusion models, Engineering Analysis with Boundary Elements, 57, 37-46, 2015.

[17] G. Yao, J. Kolibal and C.S. Chen, A localized approach for the method of approximate particular solutions, Computers and Mathematics with Applications, 61, 2376-2387, 2011.

[18] C.M. Fan, C.H. Yang and W.S. Lai, Numerical solutions of two-dimensional flow fields by using the localized method of approximate particular solutions, Engineering Analysis with Boundary Elements, 57, 47-57, 2015.

[19] A. Karageorghis and I. Kyza, Efficient algorithm for approximate particular solutions of elliptic equations using Chebyshev polynomials, Communications in Computational Physics, 2(3), 501-521, 2007.

[20] B. Khatri Ghimire, H.Y. Tian and A.R. Lamichhane, Numerical solutions of elliptic partial differential equations using Chebyshev polynomials, Computers and Mathematics with Applications, 72(4), 1042-1054, 2016.

[21] T. Dangal, C.S. Chen and J. Lin, Polynomial particular solutions for solving elliptic partial differential equations, Computers and Mathematics with Applications, 73, 60-70, 2017.

[22] C.S. Liu, A modified collocation Trefftz method for the inverse Cauchy problem of laplace equation, Engineering Analysis with Boundary Elements, 32, 778-785, 2008. 